\title{
Effect of wood filler treatment and EBAGMA compatibilizer on morphology and mechanical properties of low density polyethylene/olive husk flour composites
}

\author{
M. Kaci*, H. Djidjelli, A. Boukerrou, L. Zaidi \\ Organic Materials Laboratory, Chemical Engineering Department, Faculty of Sciences and Engineering Sciences, \\ Route de Targa-Ouzemmour, University Abderrahmane Mira, Bejaia 06000, Algeria
}

Received 28 March 2007; accepted in revised form 4 June 2007

\begin{abstract}
This paper deals with plastic-wood composites based on low density polyethylene (LDPE) and olive husk flour (OHF). The problem of incompatibility between the hydrophilic wood filler and the LDPE hydrophobic matrix was treated by two methods: a chemical modification of the olive husk flour with maleic anhydride to esterify the free hydroxyl groups of the wood components and the use of a compatibilizer agent, i. e. an ethylene-butyl acrylate-glycidyl methacrylate (EBAGMA) terpolymer. The changes in the structure, the morphology, and the properties resulting from these treatments were followed by various techniques, especially FTIR spectroscopy, scanning electron microscopy (SEM), tensile measurements and water absorption. The experimental results indicated that both methods, i.e. the chemical treatment of the olive husk flour with maleic anhydride and the inclusion of EBAGMA terpolymer, improved the interactions between the two composite components and promoted better dispersion of the filler in the matrix. Moreover, ultimate tensile properties were also increased. However, the use of EBAGMA terpolymer as compatibilizer produced better enhancement of the properties of LDPE/OHF composites compared to those treated with maleic anhydride.
\end{abstract}

Keywords: polymer composites, LDPE, olive husk, morphology, mechanical properties

\section{Introduction}

Interest in the development of new composite materials derived from wood filler and thermoplastic polymer matrices has grown markedly in the last years because there are environmental and economical advantages to producing wood flour thermoplastic composites $[1,2]$. Indeed, the composites so produced show some priorities over conventional composites such as lower density, biodegradability, lower abrasion, multifunctionality, lower cost and accessibility as renewable raw materials [3, 4]. These composites are being used in a large number of applications, including docks, window frames, and molded panel components for automotive interiors [5]. Commodity thermoplastics such as poly- ethylene (PE), polypropylene (PP), poly(vinyl chloride) (PVC), and polystyrene (PS) are the polymer matrices mostly used in the manufacture of plastic/wood filler composites [5]. Among these thermoplastics, PE represents the largest portion of the plastic/wood composites. Olive husk, one of several lignocellulosic materials, is an agricultural industrial residue produced as by-products during the olive milling process in olive-producing countries such as Algeria. Despite the advantages, use of wood flour has been restricted due to its inherent high moisture absorption capacity, thermal instability during processing, poor wettability, and poor adhesion towards polyolefins [6,7]. This weak adhesion results from the incompatibility between the hydrophilic wood filler and the hydrophobic

*Corresponding author, e-mail: kacimu@yahoo.fr

(C) BME-PT and GTE 
polymer and the difficult dispersion of wood flour in the matrix because of the intermolecular hydrogen bonding of the wood flour [8,9]. According to the literature, many attempts have been made in modifying the interfacial characteristics between the wood filler and the matrix phases [10-14]. Various surface modification techniques, like mercerisation, cyanoethylation, acetylation, coupling agent treatment, gamma ray irradiation, etc. have been reported to improve the affinity between wood flour and plastics by facilitating the flour dispersion into the polymer matrix and inducing the bond formation between the filler and the matrix $[15,16]$. The objective of this work was to investigate the modification of interfacial adhesion forces between olive husk flour and LDPE matrix in order to increase their compatibility by two different methods. The first method was based on the chemical treatment of $\mathrm{OHF}$ through esterification by reacting the hydroxyl groups of the cellulose material with maleic anhydride, whereas the second one consisted of using a coupling agent based on a terpolymer of an ethylene-butyl acrylate-glycidyl methacrylate (EBAGMA) to improve the interfacial bonding between the OHF and the LDPE matrix. The various composite materials obtained were studied by different analytical techniques as: FTIR spectroscopy, tensile properties and water absorption capacity.

\section{Experimental part}

\subsection{Materials}

The LDPE used was provided as pellets by SABIC Company from Saudi Arabia and was commercialized under the grade name of Stamylan LD 2100 NT/00. The main physical characteristics of the material are a density of $0.935 \mathrm{~g} / \mathrm{cm}^{3}$ and a melt flow index $(\mathrm{MFI})=0.6 \mathrm{~g} / 10 \mathrm{~min}$ according to ASTM D 1238/79.

The olive husk of a granular form was obtained from the olive treatment plant in the region of Bejaia in Kabylia (Algeria). Prior to any chemical modification and mixing with the LDPE, the olive husk was firstly air-dried for two weeks and ground into very fine particles. The size fraction selected after sifting the olive husk has a maximum average diameter of $40 \mu \mathrm{m}$. This is more suitable for a homogeneous dispersion in the thermoplastic matrix. The major constituents of the olive husk

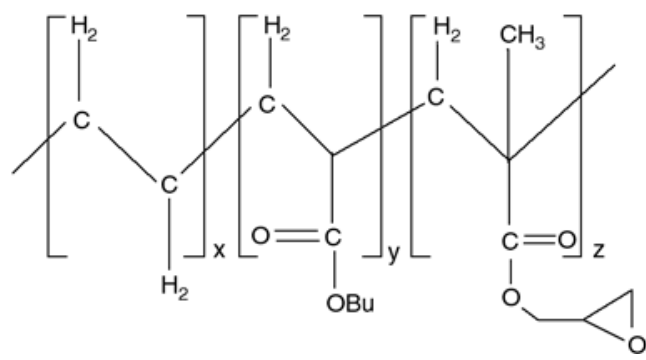

Figure 1. The chemical structure of EBAGMA terpolymer

flour were determined on the basis of absolutely dry substances using chemical procedures [17], i. e. cellulose $=39 \pm 0.5 \% \mathrm{wt}$, lignin $=20.52 \pm 0.4 \% \mathrm{wt}$, organic content $=8.5 \pm 0.8 \%$ wt and mineral filler $=$ $3 \pm 0.2 \%$ wt. The cellulose is the main constituent of the olive husk and it contains numerous hydroxyl groups that are strongly hydrophilic providing hydrogen bonds [18]. The moisture content of the filler was $=7 \pm 0.3 \%$ wt. The compatibilizer used was the terpolymer of ethylene n-butyl acrylate glycidyl methacrylate (EBAGMA) kindly supplied by DuPont (Belgium) under the trade name Elvaloy PTW. It is derived from $66.75 \%$ wt of ethylene, $28 \%$ wt of n-butyl acrylate, and $5.25 \%$ wt of glycidyl methacrylate. The chemical structure of the EBAGMA terpolymer is shown in Figure 1. The main characteristics as provided by the manufacturer, are a melt flow index $=12 \mathrm{~g} \cdot 10 \mathrm{~min}^{-1}$ as measured by ASTM method D1238, a melting point $=72^{\circ} \mathrm{C}, T_{g}=-55^{\circ} \mathrm{C}$, tensile strength at break $=5.17 \mathrm{MPa}$, and elongation at break $=950 \%$. The amount of the EBAGMA terpolymer added to the composites was fixed to $10 \mathrm{~g}$ on the basis of $100 \mathrm{~g}$ of the whole mixture of LDPE and OHF.

Other chemicals used in this study were maleic anhydride and sodium hypophosphite hydrate and xylene, all purchased from LABOSI (France). Sodium hypophosphite hydrate was used as an esterification catalyst in xylene.

\subsection{Treatment of cellulosic materials with maleic anhydride}

The modification of OHF was conducted in a reactor in the presence of a solvent. The experimental procedure used for modifying OHF was as follows [3]: $250 \mathrm{ml}$ of xylene was placed in a $500 \mathrm{ml}$ reactor, and stirred to $140^{\circ} \mathrm{C}$. After reaching this temperature, $70 \mathrm{~g}$ of maleic anhydride, $3 \mathrm{~g}$ of OHF and $1 \mathrm{~g}$ of catalyst were placed in the reactor. The reaction was carried out for two hours at $140^{\circ} \mathrm{C}$. After 
the reaction, the mixture was filtered to isolate the reacted OHF. The treated filler was soxhlet extracted with xylene for $24 \mathrm{~h}$ to remove the unreacted anhydride, and then oven-dried at $70^{\circ} \mathrm{C}$ for $24 \mathrm{~h}$. The chemical structure of the treated OHF was analyzed by FTIR spectroscopy.

\subsection{Preparation of composite samples}

Prior to mixing, the OHF was dried for 4 hours at $100^{\circ} \mathrm{C}$. Four formulations based on mixing LDPE with the wood filler before and after chemical treatment and also in the presence of the EBAGMA compatibilizer according to Table 1 were prepared in a Brabender Plasticorder, at $160^{\circ} \mathrm{C}$ and $30 \mathrm{rpm}$. Initially, LDPE was added and melted in the mixer, and then OHF and EBAGMA were introduced. The composites made out of polymer/OHF were compression moulded at $140^{\circ} \mathrm{C}$ to produce sheets of almost $3 \mathrm{~mm}$ thickness.

Table 1. Formulation codes of LDPE/OHF composites used

\begin{tabular}{|l|c|c|c|c|}
\hline $\begin{array}{c}\text { Formulation } \\
\text { codes }\end{array}$ & $\begin{array}{c}\text { LDPE } \\
{[\% \mathbf{w t}]}\end{array}$ & $\begin{array}{c}\text { Untreated } \\
\text { OHF } \\
{[\% \mathbf{w t}]}\end{array}$ & $\begin{array}{c}\text { Treated } \\
\text { OHF } \\
{[\% \mathbf{w t}]}\end{array}$ & $\begin{array}{c}\text { EBAGMA } \\
{[\mathbf{p h r}]^{*}}\end{array}$ \\
\hline LDPE & 100 & - & - & - \\
\hline LDPE/UOHF & 80 & 20 & - & - \\
\hline LDPE/TOHF & 80 & - & 20 & - \\
\hline $\begin{array}{l}\text { LDPE/UOHF/ } \\
\text { EBAGMA }\end{array}$ & 80 & 20 & - & 10 \\
\hline
\end{tabular}

*Part per hundered of the mixture LDPE/OHF

\subsection{Techniques}

\subsubsection{Fourier transform infrared spectroscopy (FTIR)}

FTIR spectra of the different composite samples were recorded using Shimadzu FTIR 8300 spectrometer using 40 cumulative scans with a resolution of $2 \mathrm{~cm}^{-1}$ within the range 600 to $3800 \mathrm{~cm}^{-1}$. The samples were analyzed using the $\mathrm{KBr}$ pellet method.

\subsubsection{Scanning electron microscopy (SEM)}

SEM was performed to investigate the morphology of the composites with a PHILIPS XL 20 instrument. The specimens were frozen under liquid nitrogen, then immediately fractured and coated with gold/palladium mixture prior to examination.

\subsubsection{Tensile test}

The different composite samples LDPE/treated and untreated OHF were subjected to tensile tests according to ASTM D 638, using Adamel Lhomargy testing machine at $50 \mathrm{~mm} / \mathrm{min}$ crosshead speed preventing the viscoelastic effect. Five measurements were conducted for each sample, and the results were averaged to obtain a mean value.

\subsubsection{Water absorption (WA)}

The water absorption was determined by measuring difference from the weight at the initial time of the test and the constant final weight of the sample according to the following procedure: the specimen dimensions for water absorption experiments were $10 \times 10 \times 3 \mathrm{~mm}$.

A minimum of three samples were tested for each material. Samples were first dried overnight at $70^{\circ} \mathrm{C}$. They were subsequently cooled in a desiccator at ambient temperature and weighted using a four-digital balance. Then, the samples were immersed in distilled water, $\mathrm{pH}=6$ and $25^{\circ} \mathrm{C}$. After 24 hours, the samples were removed and blotted to eliminate the excess water on the surface. After weighting, the water absorption (WA) of the samples was calculated as Equation (1) [16]:

$W A[\%]=\frac{M_{2}-M_{1}}{M_{1}} \cdot 100$

where $M_{1}$ and $M_{2}$ are the weights of the sample $[\mathrm{mg}]$ before and after immersion in distilled water, respectively.

\section{Results and discussion}

\subsection{FTIR analysis of the olive husk flour (OHF) before and after treatment with maleic anhydride}

Figure 2 shows the FTIR spectra recorded in the range $3600-1600 \mathrm{~cm}^{-1}$ of the OHF before and after chemical treatment with maleic anhydride which are referred to spectra (a) and (b), respectively. It is observed in spectrum (a) the presence of an absorption band localized at $1740 \mathrm{~cm}^{-1}$ which may be associated with carbonyl stretching of acetyl groups, aldehyde, carboxyl groups and esters contained in hemicelluloses, lignin and extractives. The spectrum (a) exhibits also two weak shoulders near the region $1640-1685 \mathrm{~cm}^{-1}$ which can be 


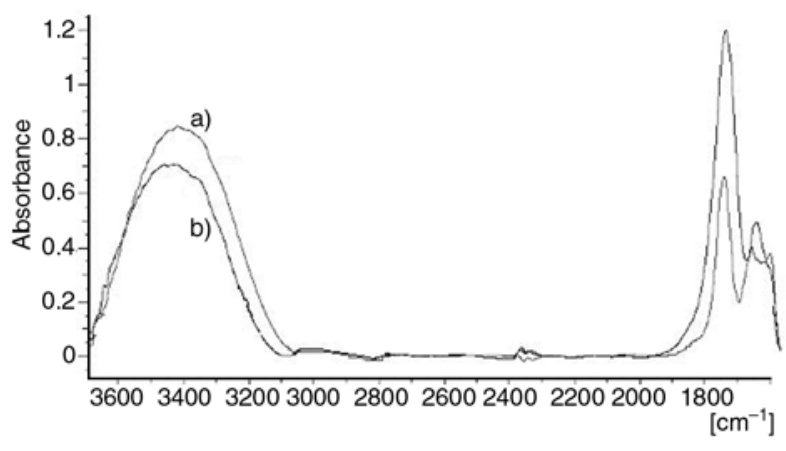

Figure 2. FTIR spectra of OHF: a) - before treatment and b) - after treatment with maleic anhydride

attributed to stretching vibrations of the $\alpha$-keto-carbonyl groups. Moreover, a large absorption band is observed at $3400 \mathrm{~cm}^{-1}$ corresponding to hydroxyl groups. After treatment as shown in spectrum (b), the absorption bands at 1740 and $1645 \mathrm{~cm}^{-1}$ both exhibit a significant growth in intensity. On the other hand, a slight decrease in the absorption band intensity of the hydroxyl groups is observed. According to the literature, these structural changes in the OHF after treatment suggest that the esterification reaction between hydroxyl groups of the OHF and anhydride groups of maleic anhydride may have occurred.

\subsection{SEM analysis of LDPE/OHF composites}

Figures 3-6 show the scanning electron micrographs of fractured samples of the neat LDPE, LDPE/UOHF and LDPE/OHF composites while using the treated filler or incorporating the terpolymer of EBAGMA as a compatibilizer, respectively. In Figure 3, the SEM micrograph of the neat LDPE reveals that no cracks are visible on the surface. In the case of untreated composite samples, illustrated in Figure 4, the micrograph shows that the addition of OHF to LDPE matrix results in a gross phase segregation morphology. OHF aggregates are formed on the surface and well defined holes

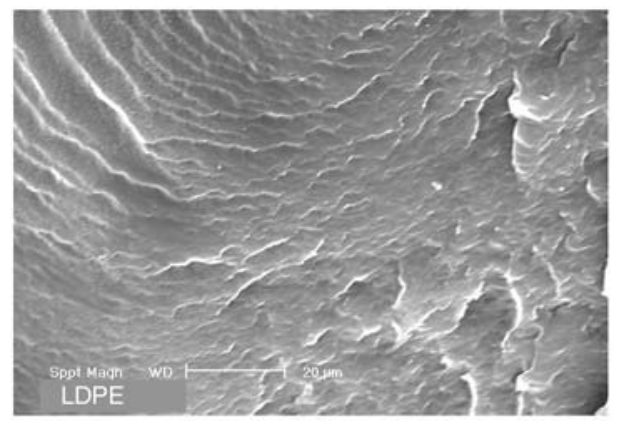

Figure 3. SEM micrographs of the neat LDPE. 500x

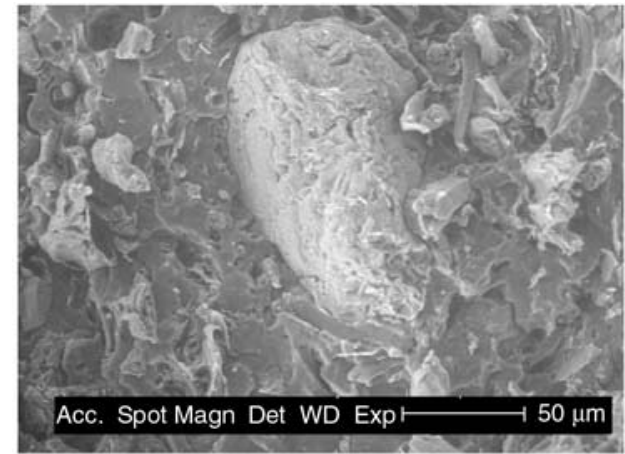

Figure 4. SEM micrographs of LDPE/untreated OHF composites. $500 x$

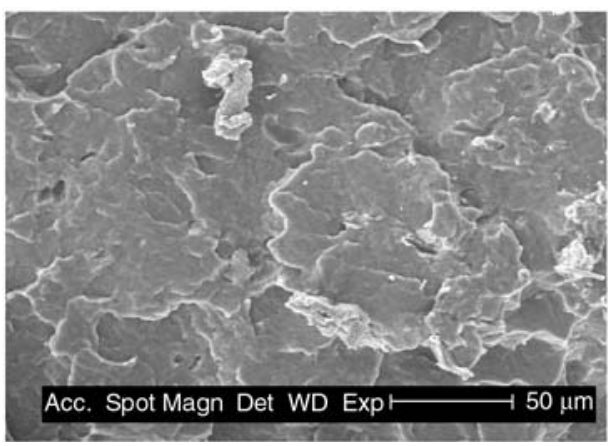

Figure 5. SEM micrographs of LDPE/treated OHF composites. $500 \times$

around the OHF particles can be seen. This is mainly due to weak interfacial interactions between the cellulosic filler and the LDPE matrix. These defects are responsible for failure and could affect the functional properties of LDPE/OHF composites. On the contrary, with the chemical treatment of OHF with maleic anhydride, the SEM micrograph in Figure 5 exhibits clearly a finer OHF dispersion. This is probably a result of chemical interactions between hydroxyl groups of OHF and maleic anhydride reducing the strong filler interactions resulting from hydrogen bonding. The addition of EBAGMA to LDPE/OHF composites improves also the dispersion of the wood filler particles in the LDPE as illustrated in Figure 6. Indeed,

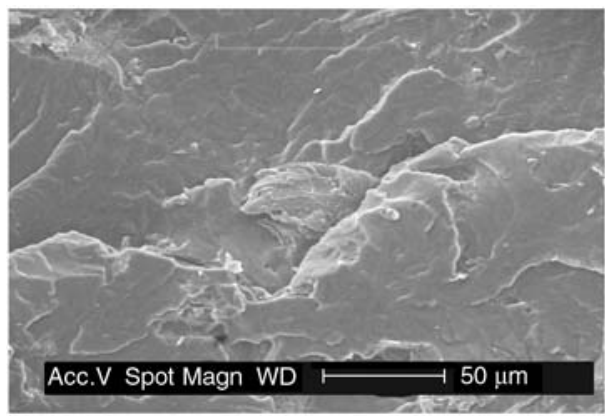

Figure 6. SEM micrographs of LDPE/untreated OHF/EBAGMA. 500× 
a decrease is observed in the size of the aggregates. This result may be attributed to the efficient activity of glycidyl methacrylate functionality of the EBAGMA terpolymer which contains both acrylic and epoxy groups to provide flexibility and adhesive strength [18].

\subsection{Mechanical properties}

Figure 7 shows the changes in the percent elongation at break for all the composite samples compared to the neat LDPE. It is evident that the addition of $20 \%$ by weight of untreated OHF to LDPE matrix leads to a sharp decrease in elongation at break from almost $326 \%$ for the neat polymer to approximately $27 \%$ for the untreated composites. As reported in literature [3], the poor dispersion of the wood filler and the reduced interfacial adhesion to the matrix may explain this decrease since the effective transfer of stress from the matrix to the filler requires an adequate interfacial adhesion. This result is in agreement with the data obtained by SEM. The chemical treatment of the filler with maleic anhydride produces a slight increase in the percent elongation at break passing from $27 \%$ for the untreated composites to $31 \%$ for the treated ones. The composites prepared using EBAGMA exhibit relatively higher value of elongation at break (almost 36\%) when compared to the maleic anhydride treated composites. In general, it is noted that the elongation at break values of the treated composites are slightly increased to those of untreated samples. This behaviour of elongation at break when the composites contain EBAGMA

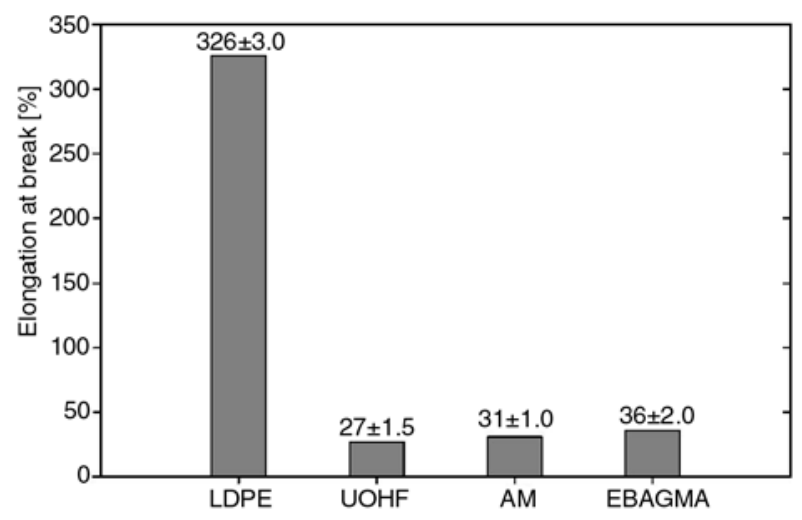

Figure 7. Percent elongation at break for LDPE/OHF composites before and after treatment compared to the neat LDPE. UOHF: untreated composites; AM: composites treated with anhydride maleic; EBAGMA: composites compatibilized with EBAGMA

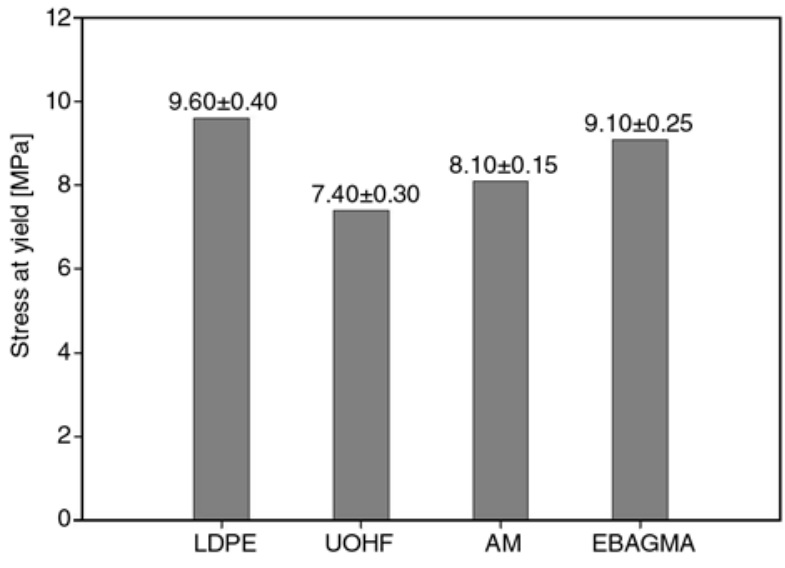

Figure 8. Stress at yield for LDPE/OHF composites before and after treatment compared to the neat LDPE. UOHF: untreated composites; AM: composites treated with anhydride maleic; EBAGMA: composites compatibilized with EBAGMA

compatibilizer is interpreted as a consequence of the acidic nature of the functionalized compatibilizer rather than to the polymer-filler interaction, because the former one accelerates degradation of cellulose fillers at the processing temperature, and subsequently leads to a brittleness of the filler particles. Figure 8 shows the values of the stress at yield of the neat LDPE and LDPE/OHF before and after treatment. The yield stress of the neat LDPE is about $9.6 \mathrm{MPa}$. With the addition of $20 \%$ of untreated OHF to the LDPE matrix, the yield stress of the composite decreases to almost 7.4 MPa. This behaviour is generally observed in non-compatible polymer composites and it can be attributed to formation aggregates by the filler particles. Subsequently, direct physical bonds between filler particles are weak and are thus easily broken during tensile testing [19]. The improved dispersion of the filler particles in the polymer matrix after treatment of OHF with maleic anhydride or addition of EBAGMA, is responsible for the increase in the yield stress. As a result, the yield stress increases to approximately 8 and $9 \mathrm{MPa}$ for LDPE/treated OHF and LDPE/UOHF/EBAGMA composites, respectively. Moreover, the general improvement in the tensile properties of the treated composites is related to an increase in compatibility between LDPE and OHF.

Figure 9 shows the effect that the chemical treatment of OHF with maleic anhydride and the EBAGMA compatibilizer have on the Young's modulus of LDPE/OHF composites. As expected, 


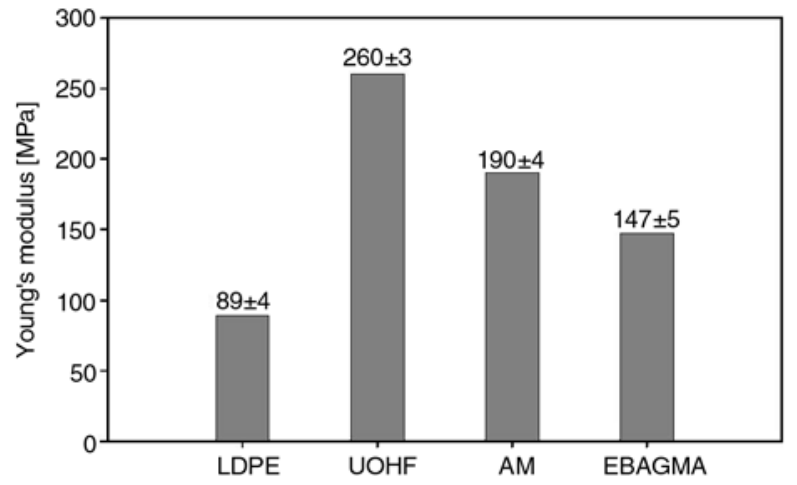

Figure 9. Young's modulus for LDPE/OHF composites before and after treatment compared to the neat LDPE. UOHF: untreated composites; AM: composites treated with anhydride maleic; EBAGMA: composites compatibilized with EBAGMA

the addition of OHF to the polymer matrix produces a significant increase in the Young's modulus passing from almost $89 \mathrm{MPa}$ for the neat LDPE to $260 \mathrm{MPa}$ for the untreated composites due to the rigid character of the filler particles. After chemical treatment of OHF with anhydride maleic, there is a considerable decrease in the Young's modulus value of the composites to almost $190 \mathrm{MPa}$ suggesting better interfacial cohesion. This decrease is more accentuated when the EBAGMA terpolymer is added to the composites reaching the value of $147 \mathrm{MPa}$. This behaviour could be explained by the resultant effect of both the acrylic functionality of the glycidyl methacrylate and the butyl acrylate group of the compatibilizer which provide better flexibility [9].

\subsection{Water absorption}

Water absorption is mainly due to hydrogen bonding of water molecules to the hydroxyl groups on the cell walls of the wood [20]. In this respect, Figure 10 shows the values of water absorption of the different LDPE/OHF composites compared to the neat polymer after $24 \mathrm{~h}$. The highest water absorption is observed for the untreated LDPE/OHF composites $(\sim 0.36 \%)$, whereas the value for pure LDPE is negligible. Both the filler treatment and the EBAGMA compatibilizer lower the water absorption of the composite samples to 0.23 and $0.19 \%$, respectively. This result could be attributed to lower amount of free $\mathrm{OH}$ in cellulose, because some of them could be interacting with the anhydride groups of anhydride maleic or glycidyl

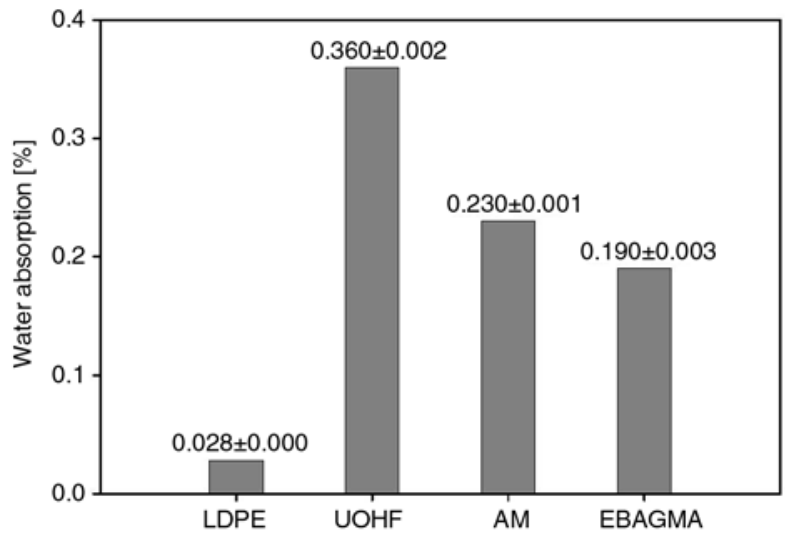

Figure 10. Water absorption for LDPE/OHF composites before and after treatment compared to the neat LDPE. UOHF: untreated composites; AM: composites treated with anhydride maleic; EBAGMA: composites compatibilized with EBAGMA

methacrylate of EBAGMA through esterification reaction confirming the FTIR data. The hydrophobic character is however more pronounced for the compatibilized composites.

\section{Conclusions}

The results show that the chemical treatment made on the OHF with anhydride maleic as well as the use of the EBAGMA terpolymer as a compatibilizer improve the dispersion of the filler particles in the LDPE matrix and produce lower water absorption compared with untreated LDPE/OHF composites. The treated composites show also a slight improvement in elongation at break and yield stress and a large decrease in tensile modulus, suggesting an increase in ductility. The improved filler dispersion and the slight increment in tensile properties induced by filler treatment or EBAGMA addition to the composites indicate an increase in compatibility between OHF and LDPE. However, the use of EBAGMA terpolymer in LDPE/OHF composites produces finer morphology, better enhancement of mechanical properties and lower water absorption when compared to the filler treatment method.

\section{References}

[1] Ichazo M. N., Albano C., Gonzalez J., Perera R., Candal M. V.: Polypropylene/wood flour composites: treatments and properties. Composite Structures, 54, 207-214 (2001). 
[2] Lee S.-H., Ohkita T.: Mechanical and thermal flow properties of wood flour-biodegradable polymer composites. Journal of Applied Polymer Science, 90, 1900-1905 (2003).

[3] Kazayawoko M., Balatinecz J. J., Matuana L. M.: Surface modification and adhesion mechanisms in woodfiber-polypropylene composites. Journal of Materials Science, 34, 6189-6199 (1999).

[4] Tserki V., Matzinos P., Panayiotou C.: Effect of compatibilization on the performance of biodegradable composites using cotton fiber waste as filler. Journal of Applied Polymer Science, 88, 1825-1835 (2003).

[5] Li Q., Matuana L. M.: Surface of cellulosic materials modified with functionalized polyethylene coupling agents. Journal of Applied Polymer Science, 88, 278286 (2003).

[6] Hristov V., Vasileva S.: Dynamic mechanical and thermal properties of modified polypropylene wood fiber composites. Macromolecular Materials and Engineering, 288, 798-806 (2003).

[7] Nunez A. J., Kenny J. M., Reboredo M. M., Aranguren M. I., Marcovich N. E.: Thermal and dynamic mechanical characterization of polypropylene-woodflour composites. Polymer Engineering and Science, 42, 733-742 (2002).

[8] Selke S. E., Wichman I.: Wood fiber/polyolefin composites. Composites: Part A: Applied Science and Manufacturing, 35, 321-326 (2004).

[9] Kaci M., Cimmino S., Silvestre C., Duraccio D., Benhamida A., Zaidi L.: Ethylene butyl acrylate glycidyl methacrylate terpolymer as an interfacial agent for isotactic polypropylene/wood flour composites. Macromolecular Materials and Engineering, 291, 869-876 (2006).

[10] Zafeiropoulos N. E., Williams D. R., Baillie C. A., Mattews F. L.: Engineering and characterization of the interface in flax fibre/polypropylene composite materials. Part I. development and investigation of surface treatments. Composites: Part A: Applied Science and Manufacturing, 33, 1083-1093 (2002).

[11] Razzino C. A., Hage Jr. E., Correa C. A.: Role of interface adhesion in wood-plastic composites. Acta Microscopica, 12, 127-128 (2003).
[12] Marcovich N. E., Aranguren M. L., Reboredo M. M.: Modified woodflour as thermoset fillers: Part I: Effect of the chemical modification and percentage of filler on the mechanical properties. Polymer, 42, 815-825 (2001).

[13] Mohanty S., Verma S. K., Nayak S. K., Tripathy S. S.: Influence of fiber treatment on the performance of sisal-polypropylene composites. Journal of Applied Polymer Science, 94, 1336-1345 (2004).

[14] Kaddami H., Dufresne A., Khelifi B., Bendahou A., Taourirte M., Raihane M., Issartel N., Sautereau H., Gérard J.-F., Sami N.: Short palm tree fibers-thermoset matrices composites. Composites: Part A: Applied Science and Manufacturing, 37, 1413-1422 (2006).

[15] Tajvidi M., Ebrahimi G.: Water uptake and mechanical characteristics of natural filler-polypropylene composites. Journal of Applied Polymer Science, 88, 941946 (2003).

[16]Malainine M. E., Mahrouz M., Dufresne A.: Lignocellulosic flour from cladodes of opuntia ficus-indica reinforced polypropylene composites. Macromolecular Materials and Engineering, 289, 855-863 (2004).

[17] Lange P. J., Mahy J. W. G.: ToF-SIMS and XPS investigations of fibers, coatings and biomedical materials. Journal of Analytical Chemistry, 353, 487493 (1995).

[18] Kaci M., Benhamida A., Cimmino S., Silvestre C., Carfagna C.: Waste and virgin LDPE/PET blends compatibilized with an ethylene butyl acrylate and glycidyl methacrylate (EBAGMA) terpolymer:1: Morphology and mechanical properties. Macromolecular Materials and Engineering, 290, 987-995 (2005).

[19] Marcovich N. E., Villar M. A.: Thermal and mechanical characterization of linear low-density polyethylene/wood flour composites. Journal of Applied Polymer Science, 90, 2775-2784 (2003).

[20] Thwe M. M., Liao K.: Environmental effects on bamboo-glass/polypropylene hybrid composites. Journal of Materials Science, 38, 363-376 (2003). 